\title{
A BOUNDARY VALUE PROBLEM IN THE HYPERBOLIC SPACE
}

\author{
P. AMSTER, G. KEILHAUER, AND M. C. MARIANI
}

Received 16 December 1999

We consider a nonlinear problem for the mean curvature equation in the hyperbolic space with a Dirichlet boundary data $g$. We find solutions in a Sobolev space under appropriate conditions on $g$.

\section{Introduction}

Let $M$ be the open unit ball in $\mathbb{R}^{3}$ of center 0 and let

$$
g_{i j}(x)=\frac{4 \delta_{i j}}{\left(1-|x|^{2}\right)^{2}}
$$

be the hyperbolic metric on $M$. Let $\Omega \subset \mathbb{R}^{2}$ be a bounded domain with smooth boundary $\partial \Omega \in C^{1,1}$, and let $(u, v)$ be the variables in $\mathbb{R}^{2}$. We consider in this paper the Dirichlet problem for a function $X: \bar{\Omega} \rightarrow M$ which satisfies the equation of prescribed mean curvature

$$
\begin{aligned}
\nabla_{X_{u}} X_{u}+\nabla_{X_{v}} X_{v} & =-2 H(X) X_{u} \wedge X_{v} \quad \text { in } \Omega, \\
X & =g \quad \text { on } \partial \Omega,
\end{aligned}
$$

where $H: M \rightarrow \mathbb{R}$ is a given continuous function, and $g \in W^{2, p}\left(\Omega, \mathbb{R}^{3}\right)$ for $1<p<\infty$, with $\|g\|_{\infty}<1$.

In the above equation $X_{u}, X_{v}$, and $X_{u} \wedge X_{v}: \Omega \rightarrow T M$ are the vector fields given by

$$
\begin{gathered}
X_{u}(u, v)=\left.\left.\sum_{k=1}^{3} \frac{\partial X_{k}}{\partial u}\right|_{(u, v)} \frac{\partial}{\partial x_{k}}\right|_{X(u, v)}, \quad X_{v}(u, v)=\left.\left.\sum_{k=1}^{3} \frac{\partial X_{k}}{\partial v}\right|_{(u, v)} \frac{\partial}{\partial x_{k}}\right|_{X(u, v)}, \\
X_{u} \wedge X_{v}(u, v)=\left.\sum_{k=1}^{3}\left(X_{u} \wedge X_{v}\right)^{k}(u, v) \frac{\partial}{\partial x_{k}}\right|_{X(u, v)},
\end{gathered}
$$

Copyright (C) 1999 Hindawi Publishing Corporation Abstract and Applied Analysis 4:4 (1999) 249-253 1991 Mathematics Subject Classification: 35J65, 53A10 URL: http://aaa.hindawi.com/volume-4/S1085337599000251.html 
where

$$
\begin{aligned}
& \left(X_{u} \wedge X_{v}\right)^{1}(u, v)=\varphi^{1 / 2}(X(u, v))\left(\left.\left.\frac{\partial X_{2}}{\partial u}\right|_{(u, v)} \frac{\partial X_{3}}{\partial v}\right|_{(u, v)}-\left.\left.\frac{\partial X_{3}}{\partial u}\right|_{(u, v)} \frac{\partial X_{2}}{\partial v}\right|_{(u, v)}\right), \\
& \left(X_{u} \wedge X_{v}\right)^{2}(u, v)=\varphi^{1 / 2}(X(u, v))\left(\left.\left.\frac{\partial X_{3}}{\partial u}\right|_{(u, v)} \frac{\partial X_{1}}{\partial v}\right|_{(u, v)}-\left.\left.\frac{\partial X_{1}}{\partial u}\right|_{(u, v)} \frac{\partial X_{3}}{\partial v}\right|_{(u, v)}\right), \\
& \left(X_{u} \wedge X_{v}\right)^{3}(u, v)=\varphi^{1 / 2}(X(u, v))\left(\left.\left.\frac{\partial X_{1}}{\partial u}\right|_{(u, v)} \frac{\partial X_{2}}{\partial v}\right|_{(u, v)}-\left.\left.\frac{\partial X_{2}}{\partial u}\right|_{(u, v)} \frac{\partial X_{1}}{\partial v}\right|_{(u, v)}\right),
\end{aligned}
$$

for $\varphi(x)=4 /\left(1-|x|^{2}\right)^{2}$.

We remark that if $X_{u}$ and $X_{v}$ are linearly independent, then $X(\Omega) \subset M$ is an imbedded submanifold and $X_{u} \wedge X_{v}(u, v)$ is the only vector orthogonal to $X(\Omega)$ at $X(u, v)$ that satisfies, for any $z=\left.\sum_{k=1}^{3} z^{k}\left(\partial / \partial x_{k}\right)\right|_{X(u, v)}$

$$
\left\langle z, X_{u} \wedge X_{v}(u, v)\right\rangle=\omega(X(u, v))\left(z, X_{u}(u, v), X_{v}(u, v)\right)
$$

where $\omega$ is the volume element of $(M,\langle\rangle$,$) , namely$

$$
\omega=\sqrt{\operatorname{det}\left(g_{i j}\right)} d x_{1} \wedge d x_{2} \wedge d x_{3}=\varphi^{3 / 2} d x_{1} \wedge d x_{2} \wedge d x_{3} .
$$

If $\nabla$ is the Levi-Civita connection associated to $\langle$,$\rangle and \Gamma_{i j}^{k}: M \rightarrow \mathbb{R}$ are the Christoffel symbols

$$
\Gamma_{i j}^{k}=\sum_{r=1}^{3} \frac{g^{r k}}{2}\left(\frac{\partial g_{r j}}{\partial x_{i}}+\frac{\partial g_{r i}}{\partial x_{j}}-\frac{\partial g_{i j}}{\partial x_{r}}\right)
$$

with $\left(g^{i j}\right)=\left(g_{i j}\right)^{-1}$, then a simple computation shows that

$$
\Gamma_{i j}^{i}(x)=\Gamma_{j i}^{i}(x)=\frac{2 x_{j}}{1-|x|^{2}}, \quad \Gamma_{i i}^{k}(x)= \begin{cases}-\frac{2 x_{k}}{1-|x|^{2}} & \text { if } k \neq i, \\ 0 & \text { otherwise }\end{cases}
$$

Let $E, F, G: \Omega \rightarrow \mathbb{R}$ be the coefficients of the first fundamental form, and the unit normal $N: \Omega \rightarrow T M$ be given by

$$
N=\frac{1}{\sqrt{E G-F^{2}}} X_{u} \wedge X_{v}
$$

which is orthogonal to the tangent space $\{X(\Omega)\}_{x}$ for any $x=X(u, v)$. Then, if $H$ : $\Omega \rightarrow \mathbb{R}$ is the mean curvature of $X(\Omega)$ we obtain

$$
\left\langle N, \frac{G}{E G-F^{2}} \nabla_{X_{u}} X_{u}+\frac{E}{E G-F^{2}} \nabla_{X_{v}} X_{v}-2 \frac{F}{E G-F^{2}} \nabla_{X_{u}} X_{v}\right\rangle=-2 H .
$$

In particular, if $X$ is isothermal, that is, $E=G, F=0$, then $\left\langle\nabla_{X_{u}} X_{u}+\nabla_{X_{v}} X_{v}, X_{u}\right\rangle=$ $0=\left\langle\nabla_{X_{u}} X_{u}+\nabla_{X_{v}} X_{v}, X_{v}\right\rangle$ and consequently

$$
\nabla_{X_{u}} X_{u}+\nabla_{X_{v}} X_{v}=-2 H X_{u} \wedge X_{v}
$$

Thus, (1.11) is the equation of prescribed mean curvature for an imbedded submanifold of $M$. 


\section{A Dirichlet problem for (1.11)}

With the notations of the previous section, we consider the Dirichlet problem (1.2). The equation of prescribed mean curvature for a surface in $\mathbb{R}^{3}$ has been studied for constant $H$ in $[3,5]$, and for $H$ nonconstant in $[1,2]$.

Without loss of generality, we may assume that $g$ is harmonic in $\Omega$. Our existence result reads as follows.

THeOREM 2.1. Let $c_{0}$ and $c_{1}$ be some positive constants to be specified. Then (1.2) is solvable for any $g \in W^{2, p}\left(\Omega, \mathbb{R}^{3}\right)$ harmonic such that

$$
\|g\|_{\infty}+2\left(c_{1}+\sqrt{c_{1}\left(c_{1}+c_{0}\right)}\right)\|\operatorname{grad}(g)\|_{2 p} \leq 1 .
$$

In the proof of Theorem 2.1, we ignore the canonical isomorphism $\partial /\left.\partial x_{k}\right|_{X(u, v)} \rightarrow e_{k}$ (with $\left\{e_{k}\right\}$ the usual basis of $\mathbb{R}^{3}$ ), and considering $X_{u}, X_{v} \in \mathbb{R}^{3}$ we may write (1.2) as a system

$$
\begin{gathered}
-\Delta X_{k}=\psi_{k}\left(X, X_{u}, X_{v}\right) \quad \text { in } \Omega, \\
X_{k}=g_{k} \quad \text { on } \partial \Omega
\end{gathered}
$$

with $\psi_{k}\left(X, X_{u}, X_{v}\right)=2 H(X)\left(X_{u} \wedge X_{v}\right)^{k}+\sum_{i, j} \Gamma_{i j}^{k}(X) \operatorname{grad}\left(X_{i}\right) \operatorname{grad}\left(X_{j}\right), 1 \leq k \leq 3$. For fixed $\bar{X} \in W_{0}^{1,2 p}\left(\Omega, \mathbb{R}^{3}\right)$ such that $\|g+\bar{X}\|_{\infty}<1$, we define $X=T \bar{X}$ as the unique solution in $W^{2, p}\left(\Omega, \mathbb{R}^{3}\right) \hookrightarrow W^{1,2 p}\left(\Omega, \mathbb{R}^{3}\right)$ of the linear problem

$$
\begin{gathered}
-\Delta X_{k}=\psi_{k}\left(\bar{X}+g,(\bar{X}+g)_{u},(\bar{X}+g)_{v}\right) \text { in } \Omega, \\
X_{k}=0 \text { on } \partial \Omega .
\end{gathered}
$$

Then, for $B=\left\{X \in W_{0}^{1,2 p}\left(\Omega, \mathbb{R}^{3}\right) \mid\|g+X\|_{\infty}<1\right\}$ the operator $T: B \rightarrow W_{0}^{1,2 p}\left(\Omega, \mathbb{R}^{3}\right)$ is well defined and a strong solution of (1.2) in $W^{2, p}$ can be regarded as $Y=g+X$, where $X$ is a fixed point of $T$. By the usual a priori bounds for the Laplacian and the compactness of the imbedding $W^{2, p}\left(\Omega, \mathbb{R}^{3}\right) \hookrightarrow W_{0}^{1,2 p}\left(\Omega, \mathbb{R}^{3}\right)$ we get the following lemma.

Lemma 2.2. $T: B \rightarrow W_{0}^{1,2 p}\left(\Omega, \mathbb{R}^{3}\right)$ is continuous. Furthermore, if

$$
C_{R_{1}, R_{2}}=\left\{X \in W_{0}^{1,2 p}\left(\Omega, \mathbb{R}^{3}\right) \mid\|g+X\|_{\infty} \leq R_{1},\|\operatorname{grad}(X)\|_{2 p} \leq R_{2}\right\}
$$

with $R_{1}<1$, then $T\left(C_{R_{1}, R_{2}}\right)$ is precompact.

Proof. For $X=T(\bar{X}), Y=T(\bar{Y})$, as $X=Y$ on $\partial \Omega$ we obtain that

$$
\begin{aligned}
\left\|\operatorname{grad}\left(X_{k}-Y_{k}\right)\right\|_{2 p} & \leq c\left\|\Delta\left(X_{k}-Y_{k}\right)\right\|_{p} \\
& =c\left\|\psi_{k}\left(\bar{X}+g,(\bar{X}+g)_{u},(\bar{X}+g)_{v}\right)-\psi_{k}\left(\bar{Y}+g,(\bar{Y}+g)_{u},(\bar{Y}+g)_{v}\right)\right\|_{p}
\end{aligned}
$$


252 A boundary value problem in the hyperbolic space

and the continuity of $T$ follows. On the other hand, if $\bar{X} \in C_{R_{1}, R_{2}}$, then

$$
\begin{aligned}
\left\|\operatorname{grad}\left(X_{k}\right)\right\|_{2 p} & \leq c\left\|\Delta X_{k}\right\|_{p}=c\left\|\psi_{k}\left(\bar{X}+g,(\bar{X}+g)_{u},(\bar{X}+g)_{v}\right)\right\|_{p} \\
& \leq \bar{c}\left(R_{2}+\|\operatorname{grad}(g)\|_{2 p}\right)^{2}
\end{aligned}
$$

for some constant $\bar{c}$ and the result follows.

Remark 2.3. By definition of $\psi_{k}$, it is clear that $\bar{c} \leq c_{1} /\left(1-R_{1}\right)$ for some constant $c_{1}$.

Proof of Theorem 2.1. With the notation of the previous lemma, by Schauder fixed point theorem, it suffices to see that $C_{R_{1}, R_{2}}$ is $T$-invariant for some $R_{1}, R_{2}$. From the previous computations, we have

$$
\|\operatorname{grad}(X)\|_{2 p} \leq \frac{c_{1}}{1-R_{1}}\left(R_{2}+\|\operatorname{grad}(g)\|_{2 p}\right)^{2} .
$$

Moreover, by Poincaré's inequality

$$
\|g+X\|_{\infty} \leq\|g\|_{\infty}+c_{0}\|\operatorname{grad}(X)\|_{2 p} .
$$

Thus, a sufficient condition for obtaining $T\left(C_{R_{1}, R_{2}}\right) \subset C_{R_{1}, R_{2}}$ is that

$$
\frac{c_{1}}{1-R_{1}}\left(R_{2}+\|\operatorname{grad}(g)\|_{2 p}\right)^{2} \leq R_{2}, \quad\|g\|_{\infty}+c_{0} R_{2} \leq R_{1} .
$$

For $R$ small enough we may fix $R_{1}=\|g\|_{\infty}+c_{0} R<1$, and then the theorem is proved if

$$
c_{1}\left(R+\|\operatorname{grad}(g)\|_{2 p}\right)^{2} \leq R\left(1-\|g\|_{\infty}-c_{0} R\right)
$$

for some $R>0$. As last condition is equivalent to our hypothesis, the result holds.

\section{Regularity of the solutions of problem (1.2)}

In this section, we state the following regularity result.

TheOREM 3.1. Let $X \in W^{1,2 p}\left(\Omega, \mathbb{R}^{3}\right)$ be a solution of (1.2). Then

(a) if $g \in W^{2, q}\left(\Omega, \mathbb{R}^{3}\right)$ for some $q>1$, then $X \in W^{2, q}\left(\Omega, \mathbb{R}^{3}\right)$,

(b) if $\partial \Omega \in C^{k+2, \alpha}, H \in C^{k, \alpha}\left(\mathbb{R}^{3}, \mathbb{R}\right), g \in C^{k+2, \alpha}\left(\bar{\Omega}, \mathbb{R}^{3}\right)$ for some $0<\alpha<1$, $k \geq 0$, then $X \in C^{k+2, \alpha}\left(\bar{\Omega}, \mathbb{R}^{3}\right)$.

Proof. (a) Let $\Delta X=f \in L^{p}$. If $p \geq q$, let $Z$ be the unique solution in $W^{2, q}$ of the problem $\Delta Z=f,\left.Z\right|_{\partial \Omega}=g$. As $\Delta(X-Z)=0$ and $X=Z$ on $\partial \Omega$ the result follows. On the other hand, if $p<q$, we obtain in the same way that $X \in W^{2, p}$. For $2 \leq p<q$ this implies that $X \in W^{1,2 q}$ and the result follows.

Now we consider the case $p<2, q$. Let $p_{0}=p$ and define

$$
p_{n+1}= \begin{cases}\frac{p_{n}^{*}}{2} & \text { if } p_{n}<2, q \\ q & \text { otherwise }\end{cases}
$$


where $p_{n}^{*}$ is the critical Sobolev exponent $2 p_{n} /\left(2-p_{n}\right)$. Then $\left\{p_{n}\right\}$ is bounded, and $X \in W^{1,2 p_{n}}$ for every $n$. If $p_{n}<2, q$ for every $n$, then $p_{n}$ is increasing and taking $r=\lim _{n \rightarrow \infty} p_{n}$, we obtain that $r /(2-r)=r$, a contradiction. Hence, $p_{n} \geq q$ or $q>p_{n} \geq 2$ for some $n$, and the proof is complete.

(b) Case $k=0$ : by part (a), choosing $q>2 /(1-\alpha)$ we obtain that $X \in W^{2, q} \hookrightarrow$ $C^{1, \alpha}\left(\bar{\Omega}, \mathbb{R}^{3}\right)$. Then $\Delta X=f \in C^{\alpha}\left(\bar{\Omega}, \mathbb{R}^{3}\right)$. By $[4$, Theorem 6.14$]$ the equation $\Delta Z=f$ in $\Omega, Z=g$ in $\partial \Omega$ is uniquely solvable in $C^{2, \alpha}\left(\bar{\Omega}, \mathbb{R}^{3}\right)$, and the result follows from the uniqueness in [4, Theorem 9.15].

The general case is now immediate, from [4, Theorem 6.19].

\section{Acknowledgement}

The authors thank Professor Jean-Pierre Gossez and the referee for their fruitful remarks.

\section{References}

[1] P. Amster and M. C. Mariani, The prescribed mean curvature equation with Dirichlet conditions, to appear in Nonlinear Anal.

[2] P. Amster, M. C. Mariani, and D. F. Rial, Existence and uniqueness of $H$-system's solutions with Dirichlet conditions, Nonlinear Anal. 42 (2000), no. 4, Ser. A: Theory Methods, 673-677. CMP 1776 298. Zbl 991.65482.

[3] H. Brézis and J.-M. Coron, Multiple solutions of H-systems and Rellich's conjecture, Comm. Pure Appl. Math. 37 (1984), no. 2, 149-187. MR 85i:53010. Zbl 537.49022.

[4] D. Gilbarg and N. S. Trudinger, Elliptic Partial Differential Equations of Second Order, 2nd ed., Fundamental Principles of Mathematical Sciences, vol. 224, Springer-Verlag, Berlin, 1983. MR 86c:35035. Zbl 562.35001.

[5] S. Hildebrandt, On the Plateau problem for surfaces of constant mean curvature, Comm. Pure Appl. Math. 23 (1970), 97-114. MR 41\#932. Zbl 181.38703.

P. Amster: Departamento de Matemática, Facultad de Ciencias Exactas y Naturales, Universidad de Buenos Aires Pabellón I, Ciudad Universitaria, 1428, Buenos Aires, ARgENTINA

E-mail address: pamster@dm.uba.ar

G. Keilhauer: Departamento de Matemática, Facultad de Ciencias Exactas y Naturales, Universidad de Buenos Aires Pabellón I, Ciudad Universitaria, 1428, Buenos Aires, ARgENTINA

E-mail address:wkeilh@dm.uba.ar

M. C. Mariani: Departamento de Matemática, Facultad de Ciencias Exactas y Naturales, Universidad de Buenos Aires Pabellón I, Ciudad Universitaria, 1428, Buenos Aires, ARgENTINA

E-mail address: mcmarian@dm.uba.ar 


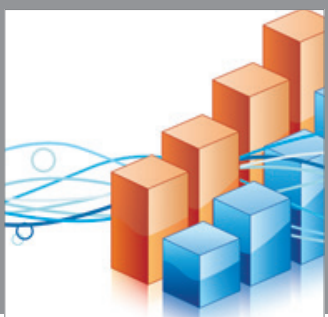

Advances in

Operations Research

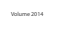

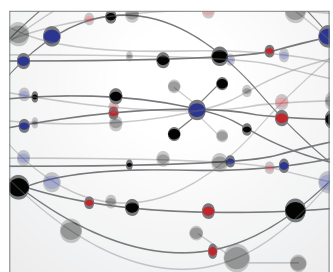

\section{The Scientific} World Journal
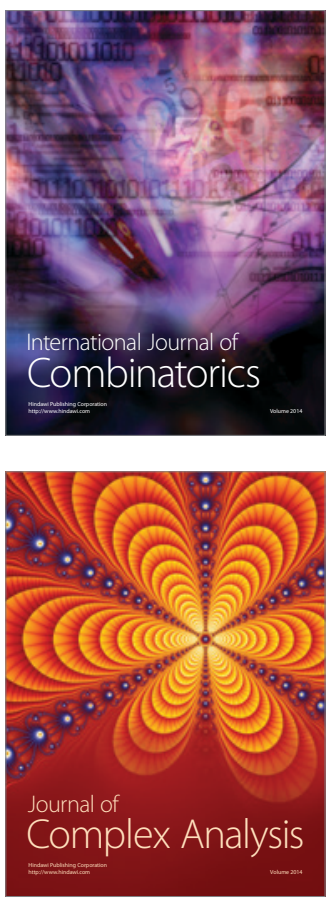

International Journal of

Mathematics and

Mathematical

Sciences
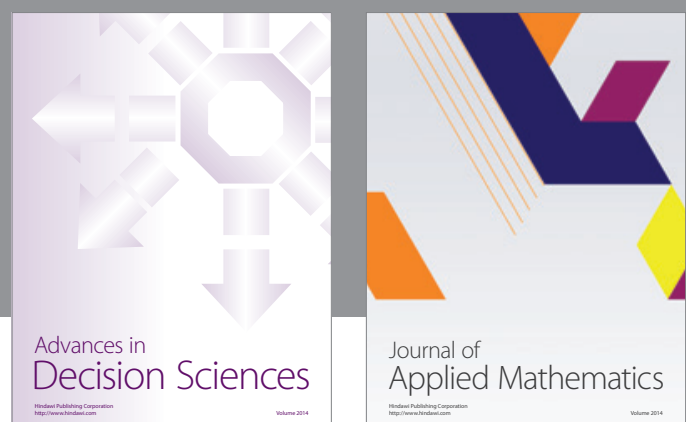

Journal of

Applied Mathematics
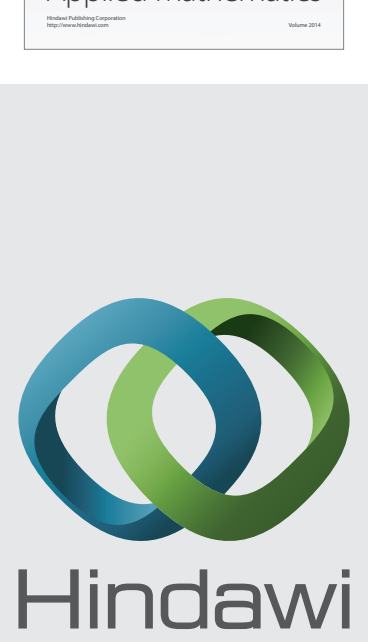

Submit your manuscripts at http://www.hindawi.com
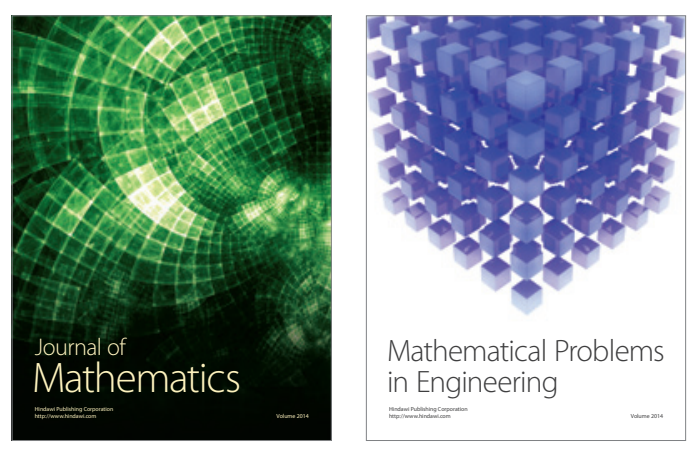

Mathematical Problems in Engineering
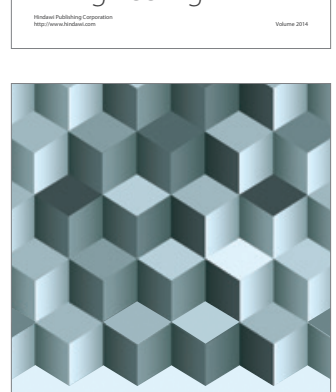

Journal of

Function Spaces
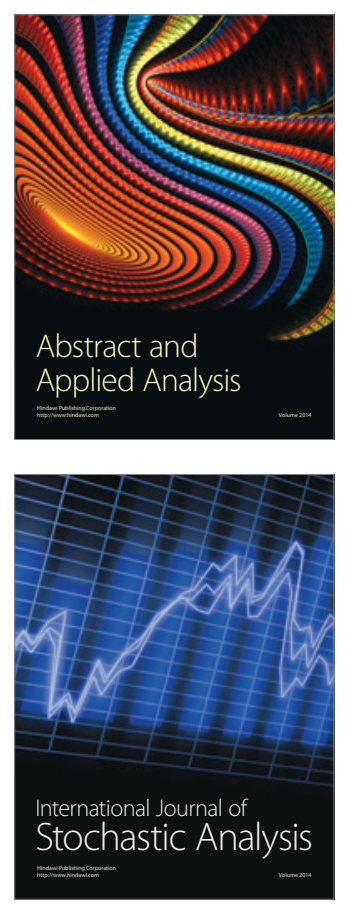

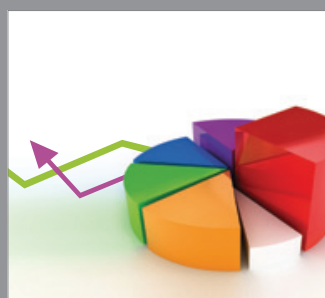

ournal of

Probability and Statistics

Promensencen
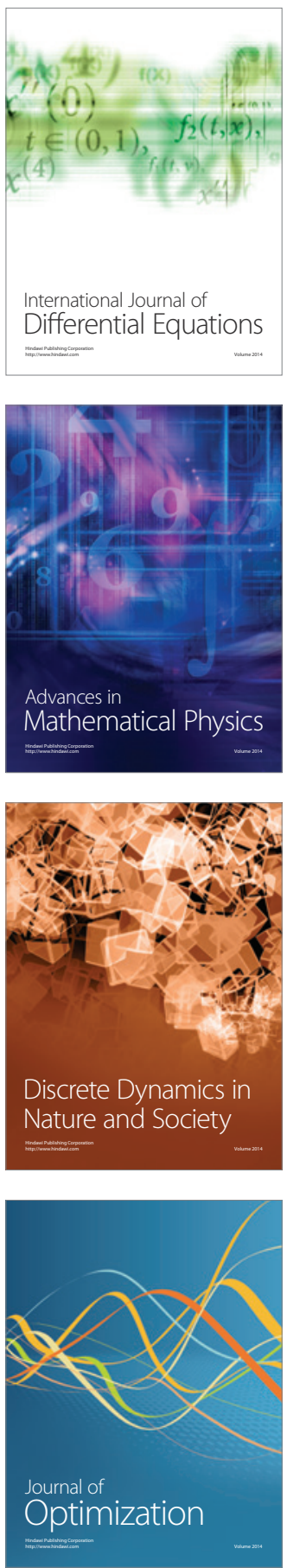